\title{
A novel image restoration technique based on anisotropic diffusion model
}

\author{
Zhao Nan $^{1 *}$ Zheng Xinqi ${ }^{1}$ Chen jianjun ${ }^{3}$ \\ ${ }^{1}$ School of Information Engineering, China University of Geosciences, Beijing, P.R.China, \\ ${ }^{2}$ School of Information Communication, Chongqing College, Chongqing, P.R.China \\ M13581920115_1@163.com, zxqsd@126.com,cjj51@sina.com
}

Keywords: image restoration; total variation; anisotropic; diffusion

Abstract. According to the principles of image restoration and non-linear constraint optimization, a novel image restoration algorithm based on the anisotropic diffusion model was proposed in this article. Based on the image textures and the gradient characteristics at the image edge, the adaptive anisotropic diffusion model was introduced and the cost function was minimized by the energy minimization scheme. Moreover, the nonlinear equations can be linearized using the fixed-point alternating iterative method. As the experimental results demonstrated, the proposed method can effectively restore the blurred images and enhance the restoration quality.

\section{Introduction}

During the acquisition process, the images are easily affected by the following factors such as the inaccurate focusing, the relative movement and the environmental influences in collecting and transmitting, all of which will lead to the reduction of imaging quality. The images will become blurred, and the noise will be added simultaneously ${ }^{[1-11]}$. In this article, to reduce the step-shaped and fuzzy phenomena in images and acquire more favorable image restoration results, we focused on the texture and structural characteristics of the blurred images, and adopted the anisotropic diffusion method to process the images, with the aim of enhancing the quality of the original images.

\section{Theoretical bases of image restoration}

Aiming at solving the problem of image blurring, Y-K method uses the joint minimization approach and the constraint conditions for $\mathrm{u}$ and $\mathrm{h}$ can be written as:

$$
\min _{\mathrm{u}, \mathrm{h}} \mathrm{J}(\mathrm{u}, \mathrm{h})=\min _{\mathrm{u}, \mathrm{h}} \frac{1}{2}\left\|\mathrm{~h} * \mathrm{u}-\mathrm{u}_{0}\right\|_{\mathrm{L}^{2}(\Omega)}^{2}+\boldsymbol{\alpha}_{1} \int_{\Omega}|\nabla \mathrm{u}|^{2} \mathrm{dxdy}+\boldsymbol{\alpha}_{2} \int_{\Omega}|\nabla \mathrm{h}|^{2} \mathrm{dx} d y
$$

Based on the Y-K method, C-W method put forward the following improvement model ${ }^{[6]:}$

$$
\min _{\mathrm{u}, \mathrm{h}} \mathrm{J}(\mathrm{u}, \mathrm{h})=\min _{\mathrm{u}, \mathrm{h}} \frac{1}{2}\left\|\mathrm{~h} * \mathrm{u}-\mathrm{u}_{0}\right\|_{\mathrm{L}^{2}(\Omega)}^{2}+\mathbf{\alpha}_{1} \int_{\Omega}|\nabla \mathrm{u}|^{2} \mathrm{dxdy}+\mathbf{\alpha}_{2} \int_{\Omega}|\nabla \mathrm{h}|^{2} \mathrm{dx} d y
$$

In this article, to keep the image smooth in the smooth regions and retain the texture details as much as possible in the texture regions, an image restoration method based on self-adaptive diffusion model was proposed, which combining the strengths of $\mathrm{C}-\mathrm{W}$ method and $\mathrm{Y}-\mathrm{K}$ method. In addition, the proposed method can reduce the noise in images as well.

\section{Image restoration based on adaptive anisotropic diffusion model}

In an image, the texture region mainly reflects its local gradient information. In this article, the joint minimization of the P-Laplace-based image restoration can be written as: 


$$
\min _{u, h} J(u, h)=\min _{u, h} \frac{1}{2}\left\|h * u-u_{0}\right\|_{L^{2}(\Omega)}^{2}+\alpha_{1} \int_{\Omega}|\nabla u|^{p(\nabla u)} d x d y+\alpha_{2} \int_{\Omega}|\nabla h|^{p(\nabla u)} d x .
$$

in which $\alpha_{1}$ and $\alpha_{2}$ are the positive parameters and $p(\nabla u)$ denotes the diffusion controlling factor, with the value range from 1 to 2 , i.e., $1 \leq p(\nabla u) \leq 2$. Here:

$$
p=\frac{2+|\nabla u|}{1+|\nabla u|} .
$$

in which the gradient $|\nabla u|$ is determined by the neighboring eight pixels around the center pixel.

a. When the gradient approaches to the infinity, $\mathrm{p} \rightarrow 1$ and the image restoration model can be converted to an anisotropic blind image restoration model by the $\mathrm{C}-\mathrm{W}$ method;

$\mathrm{b}$. When the gradient approaches to the zero, $\mathrm{p} \rightarrow 2$ and the image restoration model can be converted to an anisotropic blind image restoration model by the Y-K method.

Then we conducted the processing on Eq. (3) and take the partial derivatives with respect to $\mathrm{u}$ and $\mathrm{h}$. To simplify the calculations, the value of $\mathrm{p}$ is set and then the Euler-Lagrange Equation of Eq. (3) can be obtained. The partial derivative of Eq. (3) relative to $u$ can be expressed as:

$$
-p \alpha_{1} d i v\left(|\nabla u|^{p-2} \nabla u\right)+\left(h * u-u_{0}\right) * u(-X,-y)=0 .
$$

To take the partial derivative on Eq. (5) relative to h, the obtained Euler-Lagrange can be written as:

$$
\left(h * u-u_{0}\right) * h(-x,-y)-\alpha_{2} \nabla \cdot\left(\frac{\nabla h}{|\nabla h|}\right)=0 .
$$

Here we introduce a time variable $\mathrm{t}$ and adopt the gradient descent algorithm, the anisotropic diffusion model can be expressed as:

$$
\left\{\begin{array}{l}
u_{t}=\alpha_{1} p \nabla \cdot\left(|\nabla u|^{P-2} \nabla u\right)-\left(h * u-u_{0}\right) * u(-x,-y) \\
h_{t}=\alpha_{2} \nabla \cdot\left(\frac{\nabla h}{|\nabla h|}\right)-\left(h * u-u_{0}\right) * h(-x,-y)
\end{array}\right.
$$

To the most important diffusion term $\nabla \cdot\left(|\nabla u|^{P-2} \nabla u\right)$ in the model, we let:

$$
\frac{\partial^{2} u}{\partial x^{2}}=u_{x x}, \frac{\partial^{2} u}{\partial x \partial y}=u_{x y}
$$

In a local coordinate system, we can obtain:

$$
\frac{\partial u}{\partial t}=\nabla \cdot\left(|\nabla u|^{p-2} \nabla u\right)=|\nabla u|^{p-2} u_{\xi \xi}+(p-1)|\nabla u|^{p-2} u_{\eta \eta}
$$

in which $u_{\xi \xi}$ and $u_{\eta \eta}$ denote the two-order partial derivatives in a local coordinate system. In the above Eq. (9), the diffusion coefficients are $|\nabla u|^{p-2}$ and $(p-1)|\nabla u|^{p-2}$. 


\section{Experimental results and analyses}

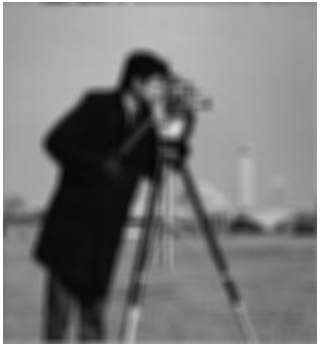

(a) Original image

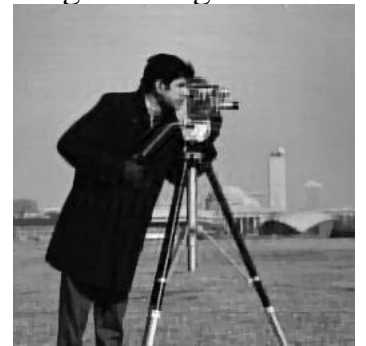

(d) Restored image using C-W method

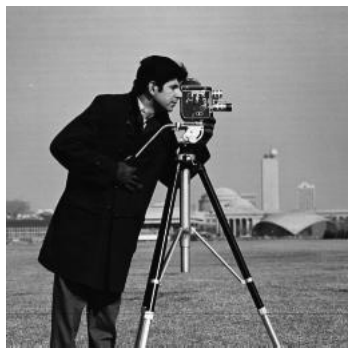

(b) Blurred image

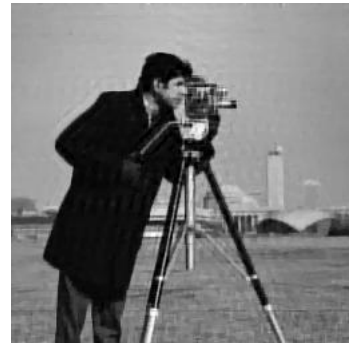

(c) Restored image using Y-K method

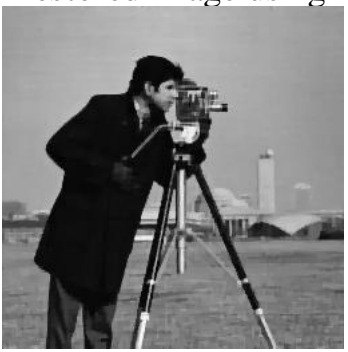

(E) Restored image using the proposed method

Fig. 1 Image restoration results using different methods

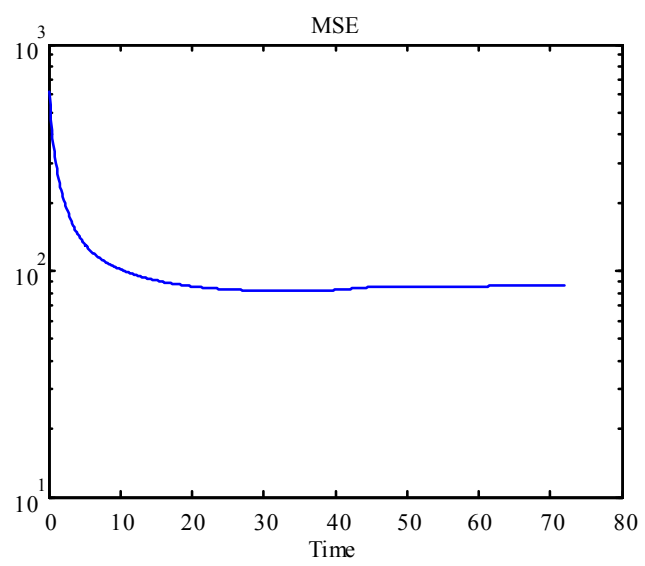

Fig. 2 Relationship between the processing velocity and the MSE of the results using the proposed method

Table 1 Comparis on of PSNR values between the blurred images and the restored images using different methods

\begin{tabular}{c|c|c|c|c}
\hline Image & $\begin{array}{c}\text { The image after the } \\
\text { addition of a } 55 \\
\text { Gaussian kernel }\end{array}$ & $\begin{array}{c}\text { The restored } \\
\text { image using Y-K } \\
\text { method }\end{array}$ & $\begin{array}{c}\text { The restored image } \\
\text { using C-W method }\end{array}$ & $\begin{array}{c}\text { The restored image } \\
\text { using the proposed } \\
\text { method }\end{array}$ \\
\hline Lena & 24.16 & 29.23 & 29.76 & 30.78 \\
\hline Peppers & 23.68 & 29.69 & 30.13 & 31.24 \\
\hline Barbara & 22.27 & 30.14 & 30.49 & 31.58 \\
\hline
\end{tabular}

\section{Conclusions}

The experimental results demonstrate that, using the proposed algorithm, the restored images can be significantly enhanced in quality, i.e., the effectiveness of the proposed method was proved.

\section{References}

[1] Zhang Jun and WeiZhi-hui. Fractional-order multiscale variation PDE model and adaptive algorithm for SAR image denoising[J]. Journal of Electronics \& Information Technology, 2010, 32(7): 1654-1659. 
[2] Inpainting images with curvilinear structures propagation[J], Machine Vision and Applications ,2014, 25:2003-2008

[3] Auto-inpainting heritage scenes: a complete framework for detecting and infilling cracks in images and videos with quantitative assessment[J] Machine Vision and Applications ,2015,26:317-337

[4] Xu Jianlou, Feng xiangchu , Hao Yan ,Improved TV-Stokes Model and Algorithm for Image Inpainting[J] Journal of Electronics \& Information Technology,2012,34(5):1142-1147

[5] Jean-François Aujol. Some first-order algorithms for total Variation based image restoration[J]. J Math Imaging Vis., 2009,34: 307-327

[6] T. F. Chan, J. Shen, and H.-M. Zhou. Total variation wavelet inpainting[J]. J. Math. Imag Vis., 2006, 25(1):107-125.

[7] Alexandru Kristaly, Hannelore Lisei, and Csaba Varga. Multiple solutions for p-laplacian type equations[J]. Nonlinear Analysis-Theory Methods \& Applications, 2008, 68(5): 1375-1381.

[8] Wu Hua, Hu Chao, WeiSui, Study of Blind Image Restoration Algorithm Based on Total VariationRegularization[J],ComputerTechnologyand Development, 2007,17(3):125-127

[9] Marcelo Bertalmio, Strong-continuation, contrast-invariant inpainting with a third-order optimal PDE[J]. IEEE Transactions on Image Processing, 2006, 15(7): 1934-1938

[10] Hong Hanyu, He Chengjian, Chen Yichao, Blind restoration algorithm of total variation based on anisotropic regularizations[J], Infrared and Laser Engineering, 2007,36(7):118-122

[11] Sun Mingzhu Zhao xin, Lu Guizhang, Semi-blind image restoration based on Canny edge detection[J], Chinese High Technology Letters, 2008,18(6):602-608. 